\title{
Shape factors of four point resistivity method in presence of rebars
}

\author{
A.J. Garzon, C. Andrade, N. Rebolledo \& J. Fullea \\ Centro de Investigación en Seguridad y Durabilidad Estructural y de Materiales (CISDEM-CSIC-UPM), \\ Madrid, Spain
}

\author{
J. Sanchez \& E. Menéndez \\ Instituto de Ciencias de la Construcción Eduardo Torroja (IETcc-CSIC), Madrid, Spain
}

\begin{abstract}
Concrete resistivity is increasingly being used for diverse applications like the detection of fibers, the evaluation of the degree of water saturation and then of the risk of rebar corrosion, the prediction of the service life or as indicator of the porosity of the concrete. There are several methods to measure the electrical resistivity of the concrete which basically are based in a direct measurement using regular geometries of the specimen and of the electrodes or the measurement by the "disc" or the four point (Wenner) method. It is said that the measurement has in any case to be made in absence of a rebar in the specimen or below the measurement place due to the presence of a metallic piece modifies the measurement. In present work is described the study made to calculate the resistivity by the Four-Point method in specimens containing a centered bar. The theoretical calculations were carried out by a finite element method and the results were verified in several specimens having the same resistivity than the numerical examples. Shape factors and a mathematical expression are given for different variables tested.
\end{abstract}

\section{INTRODUCTION}

The electrical resistivity of concrete is an indication of the volume of pores and of the degree of water saturation and therefore it has a strong relation to the concrete durability and in particular to the corrosion of reinforcements (R. B. Polder; R. Polder et al. 2000). Andrade et al. (Andrade and d'Andrea 2008) proposed a service life model based in the measurement of the resistivity in saturated concrete.

There are several methods to measure the resistivity in concrete structures. Apart from the classical or "direct" method using a regular geometry in which the electrodes are placed face to face on in a cylindrical arrangement, to relate the resistivity, $\rho$, and the electrical resistance, $R$, by the geometrical factor of the Section, $S$, and the distance between electrodes, $l$, (equation 1), there are several methods which are classified depending on the number of electrodes used.

$\rho=R \frac{S}{l}$

One method is the disc-bar method (Feliu et al. 1996). This method is based on measure the resistance between one metal electrode on the concrete surface and the reinforcement. Therefore, this method requires a connection to the reinforcement cage and to avoid being near a bar if the cover thickness is smaller than 2 times the diameter of the disc. In practice, this may require that the disc is not placed immediately over the rebars, but at some distance, for instance in the middle of the rebar mesh. The calculation of the resistivity from the measurement needs the use of a geometrical factor $(2 \pi r)$ which is given in the expression 2 where $r$ is the radius of the disc.

$\rho=2 \pi r R$

Other method is based in the use of two parallel electrodes or bars but this arrangement needs calibration for each size and shape as there is not a unique expression that could be generally applied. Finally the most common used method is the Wenner or Four-point method. This method consists of four equally spaced electrodes that are pressed onto the concrete surface. The two outer electrodes serve to apply the current and the two inner electrodes measure the difference in potential drop. The resistance is the ratio of the voltage and the current. This method has long been known and used for determining soil resistivity (Wenner 1915) and the resistance calculated from the four-point measurement can be converted to resistivity using a similar geometrical factor than the disc method but in which $a$ is equal to the electrode spacing:

$$
\rho=2 \pi a R
$$


Equation 3 is applied for homogeneous semi-infinite volumes of concrete and infinitely small electrode points (Gowers and Millard 1999; Morris et al. 1996). If the medium is finite (a specimen of limited size) it has to be considered an additional factor which will depend on the size and shape of the specimen (Morris et al. 1996). The method has been applied from long time ago but always preventing on the need to avoid to be near the metallic bars as if they are in the zone of influence of the current applied, the resistivity will be different and then not representing the concrete bulk. In present paper the disturbance is quantified experimentally and modelled by numerical analysis in order to study whether there are some functions or relations that could be established on the proportion of disturbance and then define a "bar presence" factor, $f_{b}$, in addition to the shape factor, $f_{s}$, and the geometrical factor $(2 \pi a)$ :

$\rho=f_{b} f_{s} 2 \pi a R$

\section{METHODOLOGY}

\subsection{Experimental methodology}

The specimens fabricated were of cylindrical and prismatic shape. The same mortar mix was made for all of them: water-cement (CEM I $42.5 \mathrm{R} / \mathrm{SR}$ ) ratio of 0.5 and sand-cement ratio of 3 . In some of the specimens were embedded a carbon steel B 500 $\mathrm{SD}$ bar of $8 \mathrm{~mm}$ in diameter. The specimens were cured at $20 \pm 2^{\circ} \mathrm{C}$ and $100 \%$ relative humidity in a chamber during 28 days.

The resistivity was measured by the standard arrangement of the classical Wenner Four Points method, in which the current is applied in the external electrodes and the difference in potential is measured by means of the two internal reference electrodes. The external electrodes were small pieces of reinforcing steel and the two internal ones were $\mathrm{Ag} / \mathrm{AgCl}$ reference electrodes. Currents of $1.0 \mathrm{~mA}$ were applied for the set of experiments and the voltage difference between the reference electrodes was measured every $1 \mathrm{~ms}$ after the galvanic pulse. Previously, the potential was measured during $0.1 \mathrm{~s}$ in order to find the initial condition potential. The equipment used for all the measurements was the AUTOLAB PGSTAT 30. The specimens were measured during its 28 first days.

\subsection{Finite element method}

Specimens with and without rebar were modelled with COMSOL Multiphysics Finite Element program. The AC/DC Module is used and the modelling of static electric fields is carried out using the electric potential, $V$. By combining the definition of the potential with Gauss law and the equation of continuity, it is possible to derive the classical Poisson's equation solving the following equation:

$-\nabla \cdot\left(\sigma \nabla V-J^{e}\right)=Q_{j}$

where $\sigma$ is the electric conductivity, $J^{e}$ is an externally generated current density and $Q_{j}$ is the current source.

Once the geometry was created, the boundary settings are imposed. A normal current density of 1 $\mathrm{A} / \mathrm{m}^{2}$ was imposed to one external electrode, while a ground condition was applied to the other external electrode. The difference of potential is measured between the central reference electrodes according to Wenner methodology. Several geometries were reproduced: prismatic $4 \times 4 \times 16 \mathrm{~cm}$, cylindrical $15 \times 30 \mathrm{~cm}$ or large slab $(200 \times 200 \times 200 \mathrm{~cm})$, all of them with and without rebar. For the large slab geometry it was simulated several concrete covers. The steel rebar was $8 \mathrm{~mm}$ of diameter.

The model was computed with $>300,000$ tetrahedral elements. A conjugate gradient was used as a linear system solver, and it was fixed to a $10^{-6}$ relative tolerance. The computation was carried out in desktop computer (16 cores Intel Xeon W5580 and $12 \mathrm{~Gb}$ ) and using the Spanish National Research Council cluster facilities.

\section{RESULTS}

\subsection{Experimental results}

The resistivity increases as the hydration progresses. Figure 1 shows the behaviour of one prismatic specimen without and with bar inside during the curing of concrete. The main different noticed from both responses is that, once the galvanic pulse is applied, the potential response stabilizes immediately in the specimen without bar while it is not linear in the case of embedded bars. This polarization behaviour observed when the bars are present was explained in

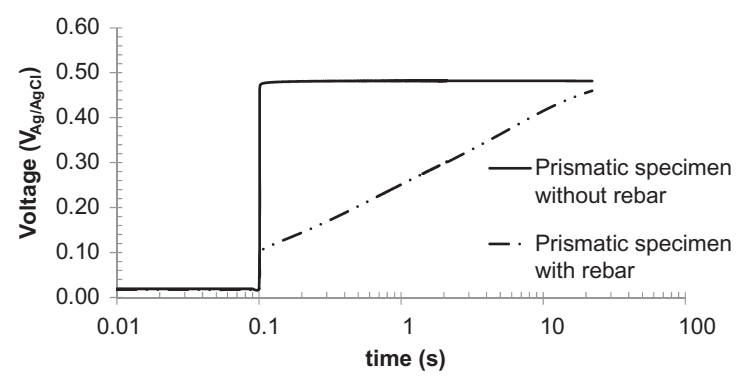

Figure 1. Experimental galvanic pulse on prismatic specimen without and with rebar at the same concrete age (1 day). 
a previous paper (Andrade et al. 2011) by the effect of the corrosion double layer existing at the steel/ concrete interface. Therefore, in order to avoid this behaviour in present tests were only the resistive part is of interest, the voltage drop is measured at $t_{g p} \rightarrow 0$, that is, for the time $=0.1 \mathrm{~s}$ according to the methodology described previously. At this time, after the current pulse, the effect of the corrosion double layer is negligible. The same behaviour is shown in Figure 2 for the case of cylindrical specimen with and without rebar. The behaviour of both specimens are different because the geometries and the concrete cover of the rebar are different in each case.

Calculating the resistivity through equation 3, a comparison is made in Figure 3 of the results obtained from the all experimental measurements. The figure shows that the results give the same values for very low resistivity values but as it increases a departure from the equality is noticeable and the differences are very dramatic from $50 \Omega \mathrm{m}$. The resistivity values in the specimen with bar does not increase anymore in spite of the increase registered in the specimen without bar.

\subsubsection{Finite element results}

Following it is shown the theoretical results for prismatic and cylindrical geometries, and after that it is shown other results on a large slab with rebar.

\subsubsection{Prismatic shape}

Figure 4 shows the theoretical results obtained for a prismatic geometry without and with rebar, $a=35 \mathrm{~mm}$ and $\rho_{\text {concrete }}=10^{3} \Omega \mathrm{m}$. The current lines spread along all geometry, but the current lines are focus between the electrodes in the rebar absence case (Figure 4 top) and they are concentrated in the rebar in the other case (Figure 4 down).

\subsubsection{Cylindrical shape.}

Same methodology is applied to cylindrical specimens. Figure 5 shows the theoretical results obtained for a cylindrical geometry without and with rebar, $a=35 \mathrm{~mm}$ and $\rho_{\text {concrete }}=10^{3} \Omega \mathrm{m}$.

Figure 6 shows the fictitious values for both geometries, prismatic and cylindrical. These theoretical results are similar than the experimental ones showed in Figure 3.

\subsubsection{Infinite shape}

Finally, the same calculations are applied to large slab with rebar of $8 \mathrm{~mm}$ diameter. The concrete cover, $r$, is changed between different simulations. Due to the symmetry of the specimen, only $1 / 2$ of the sample is simulated. The symmetry plane cuts the rebar along the axial direction. Figure 7 shows the theoretical results obtained for a large slab with rebar, $r=30 \mathrm{~mm}, a=35 \mathrm{~mm}$ and $\rho_{\text {concrete }}=50 \Omega \mathrm{m}$.

Current lines spread around all the geometry but the main current density is focussed between the electrodes. Figure 8 shows the percentage of electric current that go through the bar and the potential drop between the central electrodes versus the concrete cover. From the potential drop point of view, the bar influence disappear for concrete cover over $60 \mathrm{~mm}$.

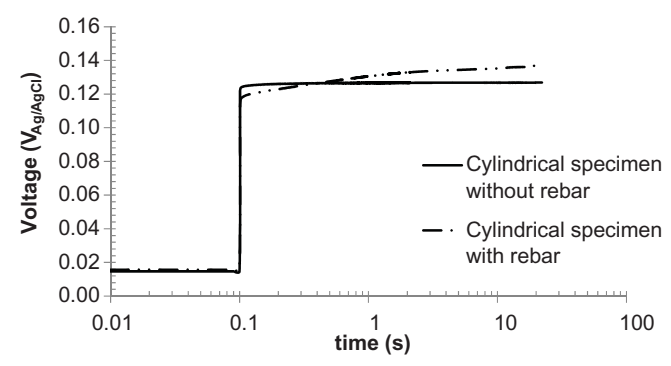

Figure 2. Experimental galvanic pulse on cylindrical specimen without and with rebar at the same concrete age (1 day).

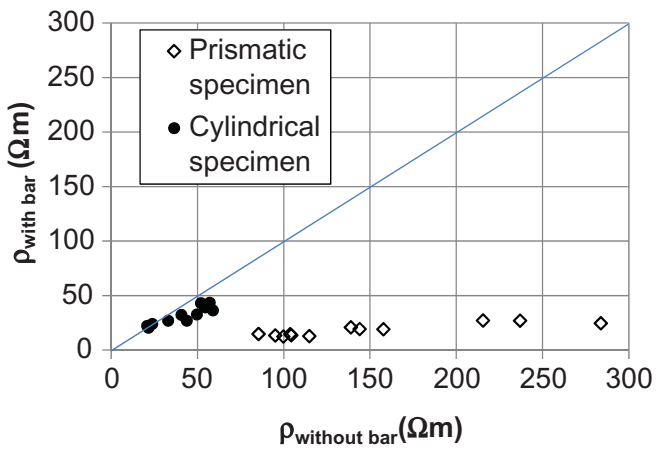

Figure 3. Experimental fictitious resistivity values according equation 3 .

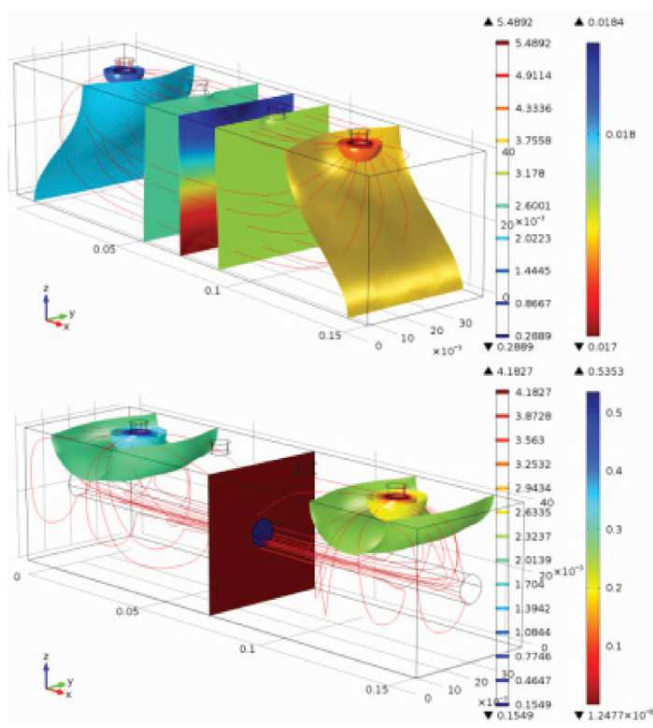

Figure 4. Prismatic geometry $4 \times 4 \times 16 \mathrm{~cm}$ without rebar (top) and with rebar (down). Isopotential surfaces, central slice of current density and current streamline distribution. FEM calculation. 


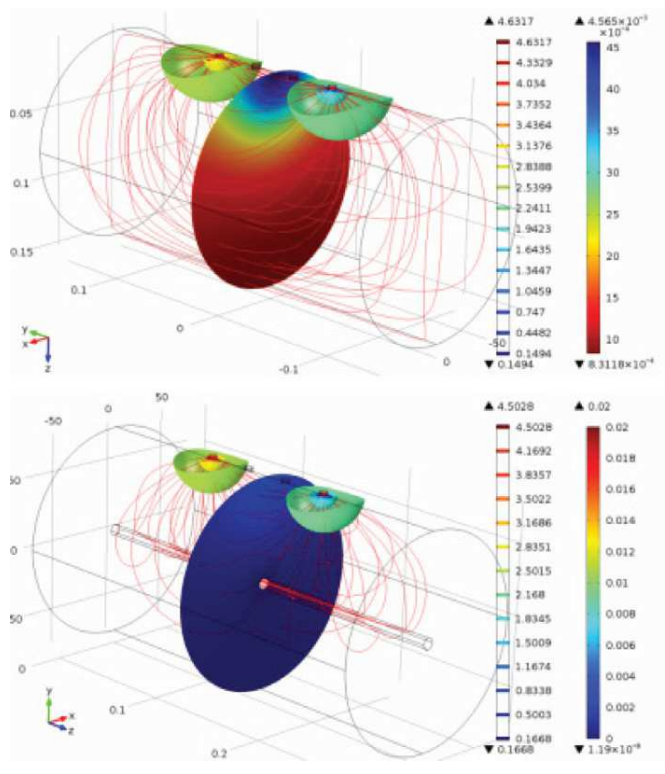

Figure 5. Cylindrical geometry $15 \times 30 \mathrm{~cm}$ without rebar (top) and with rebar (down). Isopotential surfaces, central slice of current density and current streamline distribution. FEM calculation.

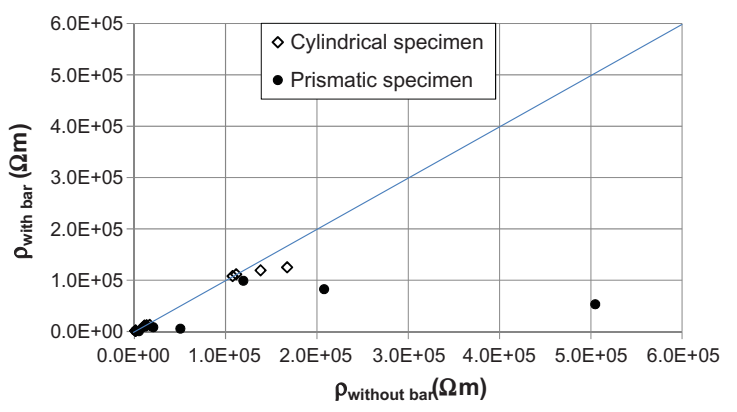

Figure 6. Prismatic and cylindrical geometry. Fictitious resistivity values according equation 3 .

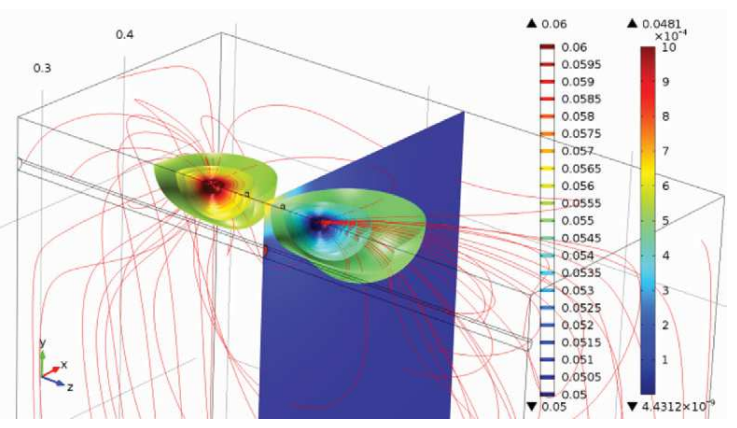

Figure 7. Large structure with $10 \mathrm{~mm}$ rebar, $30 \mathrm{~mm}$ concrete cover. Isopotential surfaces, central slice of current density and current streamline distribution. FEM calculation.

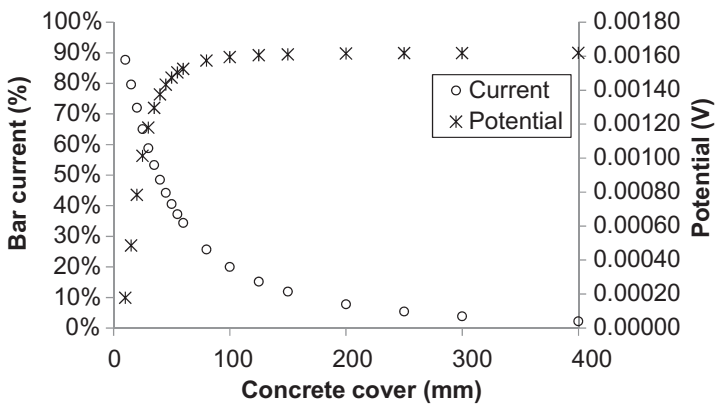

Figure 8. Percentage of electric current through the bar and the potential drop between the central electrodes versus the concrete cover.

\section{DISCUSSION}

From the results obtained in the theoretical calculations and taking into account the equation 4 , it is possible to obtain the bar presence factor, $f_{b}$, and the shape factor, $f_{s}$.

\subsection{Shape factor}

The shape factor is obtained for the prismatic and cylindrical specimen without rebar where the bar factor $f_{b}=1$. Figure 9 shows the shape factor behaviour for three different resistivity values and different electrode spacing values. It is clear that the bar factor does not depend of the resistivity.

Following equations give the shape factor in function of the electrode spacing, $a[=] \mathrm{m}$, for the prismatic and cylindrical geometry without rebar respectively:

$f_{s}=-25.006 \cdot a+1.0472$
$f_{s}=-8.5074 \cdot a+1.0282$

\subsection{Bar factor}

The bar factor is present in the prismatic and cylindrical specimens with rebar at the same time that the shape factor. Figure 10 shows the behaviour of both factors that they do not depend of the resistivity. It is remarkable that in this case $f_{b} f_{s}>1$ for all electrode spacing and resistivity values. The behaviour of the cylindrical specimen with rebar is opposite to the prismatic one, $f_{b} f_{s}<1$ for all electrode spacing and resistivity values.

Following equation shows the values of both factors in function of the electrode spacing, $a[=$ ] $\mathrm{m}$, for the prismatic and cylindrical specimen with rebar.

$$
\begin{aligned}
& f_{b} f_{s}=36.117 \cdot a+0.5945 \\
& f_{b} f_{s}=-3.3636 \cdot a+0.9618
\end{aligned}
$$




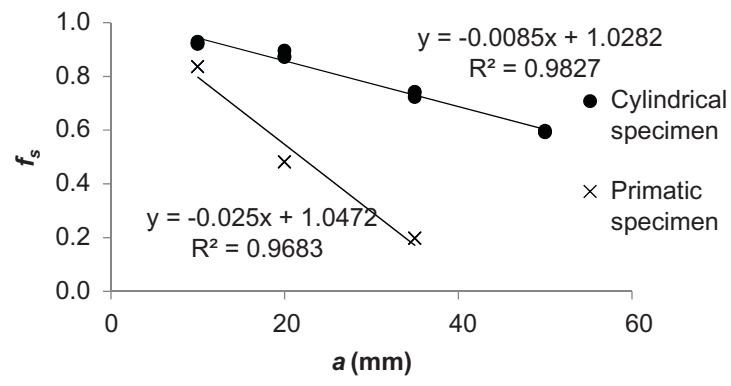

Figure 9. Relationship between the shape factor and the electrode spacing for prismatic and cylindrical geometry without rebar.

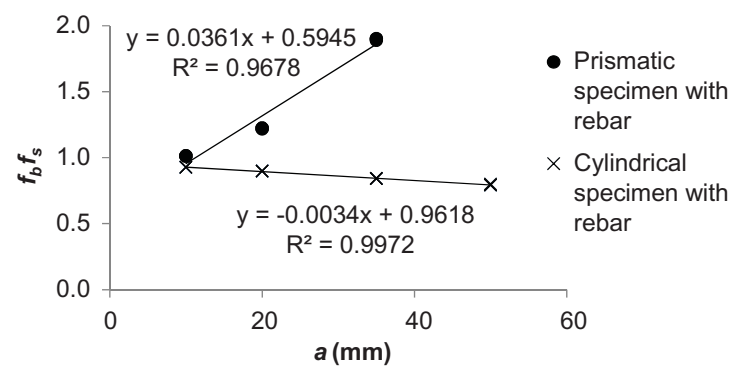

Figure 10. Relationship between the shape factor and bar factor versus the electrode spacing for prismatic and cylindrical geometry with rebar.

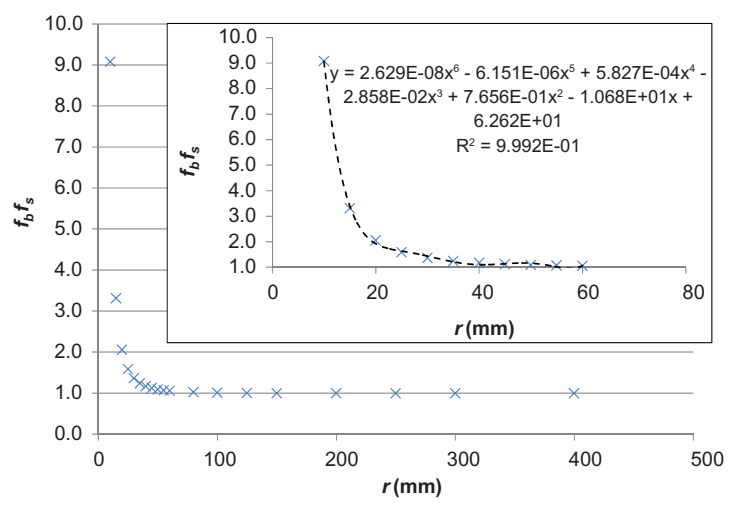

Figure 11. Geometric factor versus concrete cover for large slabs. Electrode spacing: $\mathrm{a}=35 \mathrm{~mm}$.

Figure 11 shows the evolution of the bar presence factor, $f_{b}$, and the shape factor, $f_{s}$, versus the concrete cover and the equation 10 gives the resistivity in function of the concrete cover, $r[=] \mathrm{mm}$, for the electrode spacing $a=35 \mathrm{~mm}$.

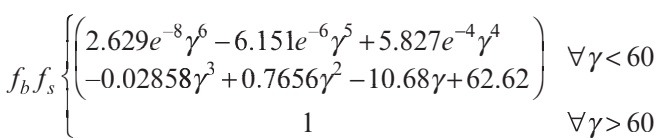

Applying the corresponding theoretical equation obtained by FEM calculations to the experimental

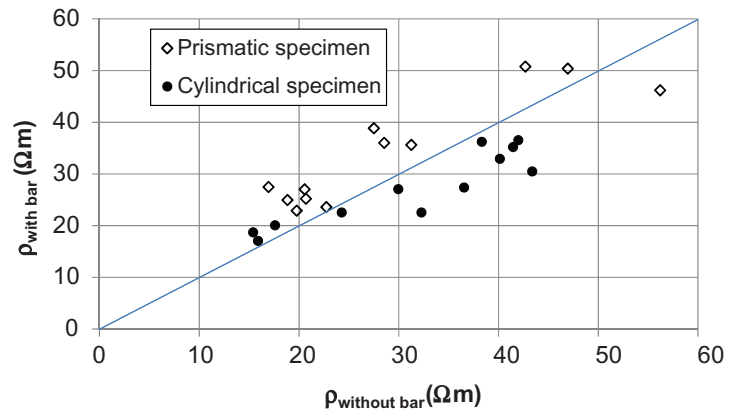

Figure 12. Experimental resistivity values for specimens with and without rebar applying the corresponding factors.

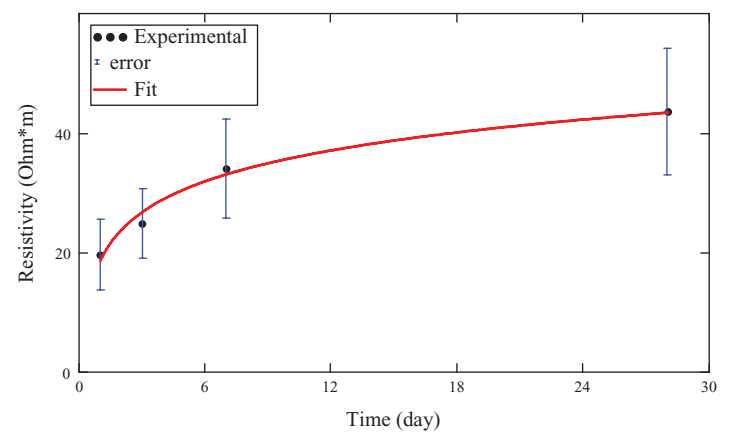

Figure 13. Resistivity evolution during the concrete aged for specimens with and without rebar.

values for each geometry, it is possible to obtain the resistivity. Figure 12 shows the resistivity values for specimens with the same dimensions with and without steel bar. The scatter data takes by the steel bar presence is reduced taking into account the proper factors. Figure 13 shows the resistivity evolution of all specimens and its variability. All the specimens belong to the same mix, so they get the same resistivity. This variability is inherent of the concrete material and it does not depend for the geometry.

\section{CONCLUSIONS}

There has been developed a methodology to measure the concrete resistivity of reinforced structures by modifying the Wenner equation. This methodology combine the experimental and the theoretical calculations of Wenner resistivity configuration.

The Wenner equation are modified in order to apply the four point configuration in a non-semiinfinite media and taking into account the bar presence effect. A shape and bar presence factors $\left(f_{s}\right.$ and $\left.f_{b}\right)$ are introduced in the Wenner equation in function of the electrode spacing and the specimen shape. In addition, it is demonstrate that these factors are independent of the resistivity itself. 


\section{ACKNOWLEDGMENTS}

This work has been financed by the Spanish MICINN: MAT2008-1497, BIA2010-18863 and INGENIO2010-CONSOLIDER Projecton "Safety and Durability of Structures: SEDUREC".

\section{REFERENCES}

Andrade, C. \& d'Andrea, R. 2008. Electrical resistivity as microstructural parameter for the calculation of reinforcement service life, in W. Sun, et al. (eds.), Microstructure Related Durability of Cementitious Composites, 1 \& 2 (Rilem Proceedings, 61; Bagneux: R I L E M Publications): 1483-90.

Andrade, C., et al. 2011. Analogue circuit of the inductive polarization resistance, Electrochimica Acta, 56(4): 1874-80.
Feliu, S., et al. 1996. A new method for in-situ measurement of electrical resistivity of reinforced concrete', Materials and Structures, 29(190): 362-65.

Gowers, K.R. \& Millard, S.G. 1999. Measurement of concrete resistivity for assessment of corrosion severity of steel using Wenner technique, Aci Materials Journal, 96(5): 536-41.

Morris, W., Moreno, E.I. \& Sagüés, A.A. 1996. 'Practical evaluation of resistivity of concrete in test cylinders using a Wenner array probe, Cement and Concrete Research, 26(12): 1779-87.

Polder, R., et al. 2000. Test methods for on site measurement of resistivity of concrete, Materials and Structures, 33(10): 603-11.

Polder, Rob B. Test methods for on site measurement of resistivity of concrete - a RILEM TC-154 technical recommendation', Construction and Building Materials, 15(2-3): 125-31.

Wenner, Frank 1915). A method for measuring earth resistivity, Journal of the Franklin Institute, 180(3): 373-75. 